\title{
Extracorporeal photopheresis practice patterns: An international survey by the ASFA ECP subcommittee
}

\author{
Nancy M. Dunbar ${ }^{1}$ | Jay S. Raval ${ }^{2}$ | Andrew Johnson ${ }^{3}$ | Cori M. Abikoff ${ }^{4}$ | \\ Jill Adamski ${ }^{5}$ | Laura L. Cooling6 | Brenda Grossman ${ }^{7}$ | Haewon C. Kim ${ }^{8}$ । \\ Marisa B. Marques ${ }^{9}$ | Shanna Morgan ${ }^{3,10,11}$ | Amy E. Schmidt ${ }^{12}$ | Steven R. Sloan ${ }^{13}$ | \\ Leon L. Su ${ }^{14}$ ｜ Zbigniew M. Szczepiorkowski1,17 | F. Bernadette West ${ }^{4}$ । \\ Edward Wong 15 | Jennifer Schneiderman ${ }^{16}$
}

${ }^{1}$ Department of Pathology and Laboratory Medicine, Dartmouth-Hitchcock Medical Center, Lebanon NH

${ }^{2}$ Department of Pathology and Laboratory Medicine, University of North Carolina, Chapel Hill, NC

${ }^{3}$ Department of Laboratory Medicine and Pathology, University of Minnesota, Minneapolis, MN

${ }^{4}$ Department of Pediatrics, New York Medical College, Valhalla, NY

${ }^{5}$ Department of Laboratory Medicine and Pathology, Mayo Clinic, Phoenix, AZ

${ }^{6}$ Department of Pathology, University of Michigan School of Medicine, Ann Arbor, MI

${ }^{7}$ Department of Pathology and Immunology, Washington University School of Medicine in St. Louis, St. Louis, MO

${ }^{8}$ Department of Pathology and Laboratory Medicine, Children's Hospital of Philadelphia, Philadelphia, PA

${ }^{9}$ Department of Pathology, University of Alabama, Birmingham, AL

${ }^{10}$ American Red Cross, St. Paul, MN

${ }^{11}$ Veterans Affairs Medical Center,

Minneapolis, MN

${ }^{12}$ Department of Pathology and Laboratory Medicine, University of Rochester Medical Center, Rochester, NY

${ }^{13}$ Department of Laboratory Medicine, Boston Children's Hospital, Boston, MA

${ }^{14}$ Department of Pathology and Laboratory Medicine, Phoenix Children's Hospital, Phoenix, AZ

${ }^{15}$ Departments of Pediatrics and Pathology, George Washington School of Medicine and Health Sciences, Division of Laboratory Medicine, Children's National Health System, Washington, DC

${ }^{16}$ Department of Pediatrics, Ann \& Robert H. Lurie Children's Hospital, Chicago, IL

\begin{abstract}
Background: Although many apheresis centers offer extracorporeal photopheresis (ECP), little is known about current treatment practices.

Methods: An electronic survey was distributed to assess ECP practice internationally.

Results: Of 251 responses, 137 met criteria for analysis. Most respondents were from North America (80\%). Nurses perform ECP at most centers $(84 \%)$ and the majority of centers treat adults only (52\%). Most centers treat fewer than 50 patients/ year $(83 \%)$ and perform fewer than 300 procedures/year (70\%). Closed system devices (XTS and/or Cellex) are used to perform ECP at most centers (96\%). The most common indications for ECP are acute/chronic skin graft versus host disease (89\%) and cutaneous T-cell lymphoma (63\%). The typical wait time for ECP treatment is less than 2 weeks (91\%). Most centers do not routinely perform quality control assessment of the collected product (66\%). There are device-specific differences in treatment parameters. For example, XTS users more frequently have a minimum weight limit $(P=0.003)$ and use laboratory parameters to determine eligibility for treatment $(P=0.03)$. Regardless of device used, the majority of centers assess the clinical status of the patient before each procedure. Greater than $50 \%$ of respondents would defer treatment for hemodynamic instability due to active sepsis or heart failure, positive blood culture in the past $24 \mathrm{~h}$ or current fever.
\end{abstract}

Conclusion: This survey based study describes current ECP practices. Further research to provide evidence for optimal standardization of patient qualifications, procedure parameters and product quality assessment is recommended.

\section{K E Y W O R D S}

apheresis, extracorporeal photopheresis, therapeutic apheresis 
${ }^{17}$ Institute of Hematology and Transfusion

Medicine, Warsaw, Poland

Correspondence

Nancy M. Dunbar, MD, Department of Pathology and Laboratory Medicine,

Dartmouth-Hitchcock Medical Center, ONE

Medical Center Drive, Lebanon, NH

03756-0001.

Email: Nancy.M.Dunbar@hitchcock.org

\section{1 | INTRODUCTION}

Although many apheresis centers offer extracorporeal photopheresis (ECP), little is known about current practice patterns. In this study, we used a survey tool to examine the scope of current ECP practices among a large cohort of respondents who represent apheresis services worldwide. The objective of this study was to describe current practices and identify areas that may warrant further research.

Extracorporeal photopheresis (ECP) is an antitumor/ immunomodulatory apheresis treatment in which leukocytes from the patient's peripheral blood are separated by centrifugation and treated with 8-methoxypsoralen (8-MOP). The latter is a naturally occurring substance that is activated following exposure to ultraviolet (UV) light resulting in DNA intercalation and ultimately apoptosis of treated cells. ${ }^{1}$ Following extracorporeal photoactivation, treated white blood cells (WBCs) are reinfused into the patient. Exactly how reinfusion of photoactivated cells results in anti-tumor and immunosuppressive effects remains incompletely understood. ${ }^{2}$ In addition to apoptosis, ECP has been shown to promote development of dendritic cells which, depending on the amount of UV exposure and interactions with other components of blood within the device, become either immature or mature dendritic cells which promote an anti-inflammatory state or an anti-tumor effect, respectively. After several months of ECP treatments, an increase in circulating regulatory T-cells and tolerogenic dendritic cells has been seen that persists following discontinuation of ECP. ${ }^{3}$

The Therakos XTS (XTS) was the first closed-system device approved for ECP by the Food and Drug Administration in 1988. This approval was granted based on a multicenter clinical trial which showed a positive response rate in patients with advanced refractory cutaneous T-Cell lymphoma (CTCL) treated with ECP. ${ }^{4}$ Approval in Europe and elsewhere around the world followed shortly thereafter. The Therakos Cellex (Cellex), a second commercially available closed system device for ECP from the same manufacturer, was approved in Europe in April 2008, Canada in January 2009, and the United States in March 2009. The XTS is a discontinuous flow instrument while the Cellex is a continuous flow instrument. Advantages of the Cellex include smaller ECV and shorter procedure times. ${ }^{5,6}$ The procedure manuals for both instruments do not specify a lower limit for patient weight, hematocrit or other laboratory parameters. Users must determine patient total blood volume and safe ECV based on patient hematocrit using tables provided in the procedure manual. There are no recommended quality control procedures to assess the collected product for either device.

At the time of our study, the most recent evidence based guidelines for ECP were those published by the American Society for Apheresis (ASFA) in 2013. ${ }^{7}$ In these guidelines, the only category I indication for ECP (first line therapy, either as primary standalone treatment or in conjunction with other modes of treatment) is erythrodermic CTCL. Category II indications (second line therapy, either as standalone treatment or in conjunction with other modes of treatment) include cellular or recurrent rejection or rejection prophylaxis in cardiac transplantation, acute/chronic skin graft versus host disease (GVHD) and lung allograft rejection/bronchiolitis obliterans syndrome. Category III indications (disorders for which the role of ECP is unestablished) include nonerythrodermic CTCL, acute/chronic non-skin GVHD, Crohn's disease, nephrogenic systemic fibrosis, pemphigus vulgaris, psoriasis and scleroderma.

\section{2 | METHODS}

An electronic survey containing 43 questions was prepared by the ASFA ECP subcommittee.

\section{1 | Center demographics}

Initial survey questions obtained the name and location of the responding center, the role of the person completing the survey, and whether or not ECP is performed at that institution.

For those centers performing ECP, subsequent questions included job classification of staff performing ECP, age range of patients treated with ECP, number of both patients and ECP procedures performed per year and devices used to perform ECP,

\section{$2.2 \mid$ General ECP practice patterns}

Subsequent questions focused on ECP practice patterns including indications for ECP, typical wait time for treatment, barriers to receiving ECP treatment, types of venous 
access used, and quality control assessment of the product collected for UV photoactivation.

\section{3 | Device-specific practices}

General practice questions were followed by questions about device-specific ECP practices including patient weight limitations, volume prime practices, laboratory or clinical findings that would prevent ECP from being performed and anticoagulant(s) used.

\section{4 | Survey development, distribution and analysis}

The survey was developed by members of the ASFA ECP subcommittee. Prior to general circulation, the survey was "beta-tested" by apheresis staff (physicians and nurses) not on the ECP committee to assess clarity of questions and time required to complete the survey.

An internet-based tool (Survey Monkey, Palo Alto, CA, www.surveymonkey.com) was utilized to administer the survey which was sent via email to over 5000 possible participants in the United States and abroad using the ASFA electronic distribution lists. Given the breadth of the ASFA membership, it is likely that more than one person received the survey at each institution but only one response per institution was retained for analysis. When multiple responses were received, the one selected for data analysis was chosen based on the respondent's role according to the hierarchy of Medical Director, Supervisor, Nurse, Physician and Medical Technologist. Responses were collected over a one-month time period (April 2015) and analyzed using descriptive statistics. Pediatric patients were defined as those under 18 years of age.

Percentages for individual survey items were calculated based on the number of responses received for each question. Categorical data were analyzed using Fisher's exact test to calculate a two-tailed $P$ value. For continuous data, an unpaired $t$ test was performed to compare means. A $P$ values of less than 0.05 was considered statistically significant. All analyses were done with web-based software ( GraphPad QuickCalcs, La Jolla, CA, www.graphpad.com).

\section{3 | RESULTS}

\section{1 $\mid$ Center demographics}

The study yielded 251 responses, of which 56 were excluded as duplicates, seven were excluded because they contained insufficient information to determine whether or not they were duplicates, and 51 because they were from centers that do not perform ECP (Figure 1). The remaining 137 evaluable responses came primarily from centers in North America and Europe (Figure 2). Center demographic information is shown in Table 1.

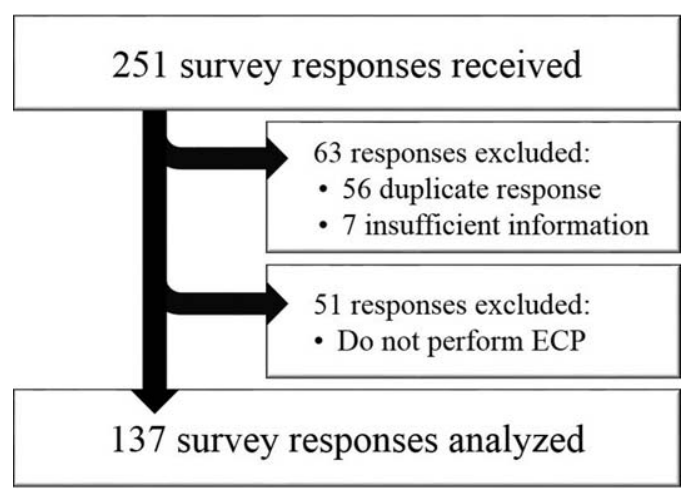

FIGURE 1 Survey total number of responses, exclusions and final number of responses analyzed

\section{2 | General ECP practice patterns}

ECP is most frequently performed for acute/chronic GVHD with skin involvement followed by CTCL and acute/chronic GVHD with non-skin involvement. A minority of centers are performing ECP for uncategorized indications per the 2013 ASFA guidelines. ${ }^{7}$ Center responses for indications for ECP and 2013 ASFA categorization are shown in Table 2. Data on typical wait time for treatment, barriers to receiving ECP treatment, types of venous access used, performance of quality control and quality control parameters are shown in Table 3.

\section{3 | Device-specific practices}

Centers using the Cellex and/or XTS devices provided additional device specific practice information shown in Table 4. Although the majority respondents indicated that their center has a minimum weight limit to enable treatment on the XTS device, most do not require a minimum weight for treatment on the Cellex device $(P=0.003)$. Among centers that provided their minimum weight requirements, the cut-offs varied widely both within and between the different devices used

\section{Geographic Distribution of Survey Responses} $(\mathrm{n}=137)$

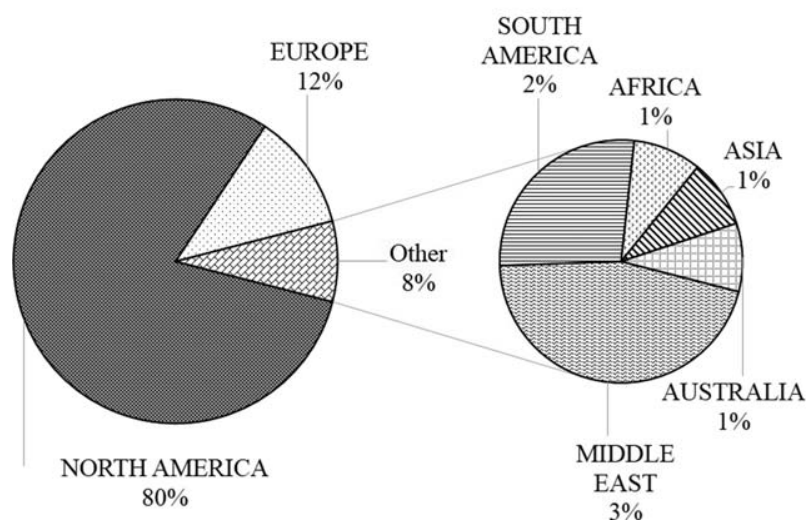

FIGURE 2 Survey responses (\%) by geographic location 
TABLE 1 Demographics of centers performing ECP

Question $\quad N(\%)$

What is the job classification of staff performing ECP at your center? $(N=130)$

Nurses only $109(84 \%)$

Both nurses and medical technologists $18(14 \%)$

Medical technologists only

What is the age range of patients treated with ECP at your center? $(N=130)$

$\begin{array}{ll}\text { Adult Only ( }>18 \text { years }) & 67(52 \%) \\ \text { Both Adult and Pediatric } & 54(41 \%) \\ \text { Pediatric Only }(0-18 \text { years }) & 9(7 \%)\end{array}$

Approximately how many patients are treated with ECP at your center per year? $(N=130)$

$\begin{array}{ll}1-20 & 80(62 \%) \\ 21-50 & 28(21 \%) \\ >50 & 22(17 \%)\end{array}$

Approximately how many ECP procedures do you perform at your center per year? $(N=130)$

$\begin{array}{ll}50-150 & 45(35 \%) \\ 151-300 & 46(35 \%) \\ 301-450 & 8(6 \%) \\ >450 & 31(24 \%)\end{array}$

Which device(s) are used at your center to perform ECP? $(N=115)$

$\begin{array}{ll}\text { Cellex and XTS } & 42(37 \%) \\ \text { Cellex ONLY } & 35(30 \%) \\ \text { XTS ONLY } & 19(17 \%) \\ \text { XTS and Off-line } & 8(7 \%) \\ \text { Cellex and Off-line } & 6(5 \%) \\ \text { Off-line system ONLY } & 5(4 \%)\end{array}$

(Figure 3). The average minimum weight for treatment on the Cellex was $27 \mathrm{~kg}$ compared to an average minimum weight of $36 \mathrm{~kg}$ for treatment on the XTS $(P=0.0095)$.

Although the majority of responding centers indicated that the decision to perform ECP is influenced by patient laboratory parameters, regardless of the device used, a higher percentage of XTS users reported using laboratory test results to determine eligibility for ECP when compared to Cellex users $(P=0.03)$. Laboratory values commonly assessed to determine eligibility for ECP include hematocrit, platelet count and WBC count. There is little agreement on the cut-off used for these assays (Figure 4).

\section{4 | DISCUSSION}

This report represents the first published survey to specifically assess ECP practice patterns by apheresis providers. We believe that it contains important information to guide future research. We found that the majority of centers performing ECP treat small numbers of patients. This observation is significant because it represents a model which in general lends to greater variation, lower adherence to protocols, and increased complications and cost. ${ }^{8-10}$ However, we also observed that center staffing, number of devices and distance to the center are barriers to care reported by up to a quarter of participating ECP centers. This suggests that access to ECP is problem for some patients and small centers are necessary to increase patient access to treatment.

The majority of respondents utilize ECP in patients with acute/chronic skin GVHD and CTCL, which are assigned Category II and I indications, respectively in the 2013 ASFA guidelines. ${ }^{7}$ Interestingly, we confirmed that a small number of practitioners are also utilizing ECP for several indications that have not been assigned a category recommendation in the 2013 ASFA guidelines including rejection following kidney and liver transplant, ulcerative colitis and other inflammatory bowel disease. Since conducting this survey, updated ASFA guidelines have been published. The only notable change to the category recommendations for ECP shown in Table 2 is the change in non-skin GVHD from category III to category II. ${ }^{11}$ Further research into the efficacy of ECP for conditions that remain uncategorized, and for which ECP is being performed at some centers, is warranted.

Although quality control assessment of the ECP product is not required, the survey revealed that $34 \%$ of responding centers routinely perform quality control testing on the cellular product prior to re-infusion. The survey was not designed to assess whether quality control results are utilized in making clinical decisions or alterations in the actual procedure. However, in the setting of a prospective study, the evaluation of the collected product could potentially be predictive of response to therapy and overall outcome of patients. Further evaluation of this topic may contribute to improvement in understanding of the mechanism of action of ECP.

Current choices for performing ECP are limited to three options: the Cellex system, the XTS system, or an off-line system. The majority of centers deliver ECP using one or both of the approved closed systems while a few centers use the off-line system. The ease of the integrated system and reduced risks for infection and infusion errors likely contribute to the predominant use of closed systems.

The Cellex and the XTS systems offer different features in relation to the extracorporeal volume, treatment time, and other parameters, and as a result their use in practice 
TABLE 2 Indications for ECP

\begin{tabular}{lll}
$\begin{array}{l}\text { In the past } \mathbf{1 2} \text { months, for which indications has ECP been } \\
\text { performed at your center? (select all that apply) }(\mathbf{N = 1 2 2})\end{array}$ & ASFA category* \\
\hline Graft versus-host disease, Skin (acute/chronic) & $108(89 \%)$ & II \\
Cutaneous T-Cell lymphoma & $79(63 \%)$ & I \\
Graft versus-host disease, Non-skin (acute/chronic) & $77(63 \%)$ & III \\
Solid Organ Transplant Rejection, Lung & $53(43 \%)$ & II \\
\hline Solid Organ Transplant Rejection, Heart & $26(21 \%)$ & II \\
\hline Scleroderma & $9(7 \%)$ & III \\
Nephrogenic Systemic Fibrosis & $6(5 \%)$ & III \\
Solid Organ Transplant Rejection, Kidney & $5(4 \%)$ & NC \\
Crohn's Disease & $5(4 \%)$ & III \\
\hline Solid Organ Transplant Rejection, Liver & $3(2 \%)$ & NC \\
\hline Pemphigus Vulgaris & $3(2 \%)$ & III \\
Other Inflammatory Bowel Disease & $2(2 \%)$ & NC \\
\hline Ulcerative Colitis & $1(1 \%)$ & NC \\
Psoriasis & $0(0 \%)$ & III
\end{tabular}

*See reference 7.

demonstrates notable differences. The majority of centers require a minimum weight limit for patients when using the XTS. This is in contrast to the use of the Cellex, in which the majority of centers do not specify a minimum weight limit. The Cellex system offers the ability to perform dual needle procedures further decreasing the run time and extra corporeal volumes when compared to the XTS or even single needle on the Cellex. ${ }^{12}$ Due to the discontinuous flow and extracorporeal volume required while performing ECP procedures on the XTS system, it can be technically challenging on smaller weight patients. ${ }^{13}$

Published reports have demonstrated the safety of the Cellex in the pediatric patient population, and it has even been shown to be better tolerated. ${ }^{5,6}$ In order to accommodate lower weight patients, a volume prime or fluid bolus can be administered. The XTS requires a larger extracorporeal volume potentially warranting a fluid prime to avoid hypotensive reactions, and when both options are available the Cellex may preferentially be selected to treat smaller patients who would be more likely to need a prime in general.

The majority of responding centers also indicated that they have laboratory thresholds that would defer the performance of ECP. XTS users more frequently rely on laboratory parameters to assess eligibility for ECP. The most common laboratory parameter reported to impact the decision to perform ECP was patient pre-procedure hematocrit, with a threshold in the range of $<24-28 \%$ being the most frequent. While not based on ECP- specific safety data, this is not altogether surprising given that the extracorporeal volume for these procedures is 220 to $620 \mathrm{ml}$ for the XTS (depending on bowl size used) and 216-266 $\mathrm{ml}$ for the Cellex (double needle vs. single needle procedure). Intraprocedure hematocrits in patients have been demonstrated to decrease by up to 9\% while on apheresis instruments, and maintenance of an intraprocedure hematocrit $>24 \%$ appears to be compatible with evidence-based transfusion guidelines for stable, non-critically ill patients. ${ }^{14,15}$

There was less consensus surrounding the minimum platelet count for performance of ECP with a range of platelet count from $20-50,000 / \mathrm{mcl}$ that would prevent a patient from receiving ECP. This again appears to be based not on ECP-specific safety data but rather on extrapolation from platelet transfusion thresholds in patients undergoing interventional procedures or surgeries. ${ }^{16}$ It is interesting that the XTS users tend to favor higher platelet counts even though the Cellex appears to remove more platelets from peripheral circulation during collection of buffy coat when compared to the XTS. ${ }^{17}$

With regard to WBC, almost half of respondents indicated that WBC had to be $>1000 / \mu 1$ for either the XTS or Cellex. Though there is still no data indicating what is an adequate treatment dose of buffy coat and how that relates to peripheral WBC count, this reported WBC threshold is a reasonable one based on extrapolations of patient data from ECP-specific literature regarding collection efficiency kinetics and clinical outcomes. ${ }^{17,18}$ 
TABLE 3 General ECP practices

$\begin{array}{lc}\text { Question } & N(\%) \\ \text { What is the typical wait time at your center between } \\ \text { the initial referral and the first ECP procedure? }(\mathrm{N}=122) \\ \text { Less than one week } & 61(50 \%) \\ 1-2 \text { weeks } & 50(41 \%) \\ 3 \text { or more weeks } & 11(9 \%)\end{array}$

Which of the following are barriers to patients receiving ECP treatment at your center? (select all that apply) $(N=122)$

\begin{tabular}{ll} 
Venous access & $71(58 \%)$ \\
\hline Insurance coverage & $61(50 \%)$ \\
Staffing & $34(28 \%)$ \\
Number of devices & $30(25 \%)$ \\
Distance to center & $26(21 \%)$
\end{tabular}

Which types of venous access are used or considered acceptable for performing ECP at your center? (select all that apply) $(N=122)$

\begin{tabular}{ll} 
Tunneled catheter & $109(89 \%)$ \\
\hline Peripheral IV & $103(84 \%)$ \\
\hline Vortex/Power port & $77(63 \%)$ \\
High flow PICC & $13(11 \%)$
\end{tabular}

Do you routinely perform quality control assessment of the collected ECP product using any laboratory parameters? $(N=122)$

Yes $41(34 \%)$

No

$80(66 \%)$

If you routinely perform quality assessment, which of the following laboratory parameters on the collected ECP product are routinely assessed? (select all that apply) $(N=41)$

\begin{tabular}{ll} 
Total cell count & $34(83 \%)$ \\
\hline Hematocrit & $31(76 \%)$ \\
\hline Lymphocyte count & $30(73 \%)$ \\
\hline Monocyte count & $25(61 \%)$ \\
\hline Bacterial culture & $10(24 \%)$ \\
\hline Flow cytometric assay & $5(12 \%)$ \\
\hline Apoptosis assay & $4(10 \%)$ \\
\hline Evaluation of cell populations & $3(7 \%)$ \\
Proliferation assays & $37 \%)$ \\
Cytokine levels & $1(2 \%)$
\end{tabular}

Regardless of instrument used, the most common anticoagulant used is heparin alone or heparin and/or ACD-A. There are protocols available to use only ACD-A as the anticoagulant during the apheresis, and a few of the reporting centers use ACD-A exclusively. ${ }^{19}$ Employing these protocols can avoid the systemic anticoagulation effects of heparin, and are necessary when treating patients with contraindications to heparin.

While no absolute clinical contraindications to performance of ECP exist (aside from aphakia and idiosyncratic reactions associated with 8-MOP), the majority of responding centers indicated that patient clinical status does impact the decision to perform ECP. In over $90 \%$ of respondents, hemodynamic instability due to sepsis or heart failure would result in the cancellation of ECP. Patients with positive blood cultures or fever were additional indications for which ECP procedures would be cancelled for about half of responding institutions. Although other types of apheresis procedures have been shown to be tolerable and feasible in critically ill adult and pediatric patients, ECP regimens are long-term therapies performed on an outpatient basis. ${ }^{20-22}$ Thus, the performance of ECP under situations of hemodynamic instability or critical illness, presents an unfavorable risk-benefit ratio. The fact that these clinical concerns are not universally shared by all institutions indicates clinical equipoise and the need for larger studies to clarify appropriate clinical contraindications for performing ECP.

As for any survey-based study, limitations in our study exist including errors in coverage (i.e., not everyone who performs ECP received the survey), sampling, non-response, and measurement (i.e., misinterpretation of questions or providing different answers for a theoretical survey versus actual clinical practice). Second, the current study's findings primarily reflect the practice patterns in North America, specifically the United States. As such, the patient populations, ECP instrumentation, and clinical practice patterns cannot necessarily be generalized to other countries.

Multiple responses may have been received from the same institution and a hierarchy based on the role of the person completing the survey was used to select the response used for data analysis. The hierarchy prioritized the Medical Director which may not have worked equally well for all survey questions. Further, when results were received from the same institution they were not compared to ensure that responses were the same for all survey questions. In addition, survey data were not weighted based on the number of patients at each institution. As a result, each responding institution's data were counted equally which may skew the data in favor of practices reported by small volume centers.

This survey was not designed to address differences between ECP in pediatric versus adult patients. The pediatric population brings many unique considerations to the performance of ECP, including procedural modifications based on small body mass/total blood volumes, circuit priming/ fluid boluses, anticoagulation, ability to tolerate extracorporeal procedures, and venous access issues. Future research 
TABLE 4 Device specific practices

$\begin{array}{llll}\text { Question } & \text { XTS } & \text { Cellex } & P \text { values }\end{array}$

Is there a lower limit for patient weight required for the use of this instrument at your center? (XTS =69; Cellex $=82$ )

Yes

$41(60 \%)$

$28(34 \%)$

0.003

No

$28(40 \%)$

$54(66 \%)$

Does your center ever perform volume prime or administer fluid boluses for lower weight patients when using this instrument? (XTS $=68$; Cellex $=80$ )

$\begin{array}{llll}\text { Yes } & 28(41 \%) & 44(55 \%) & \text { NS } \\ \text { No } & 40(59 \%) & 36(45 \%) & \end{array}$

Is the decision to provide ECP treatment at your center using this instrument dependent on any patient laboratory parameters? $(\mathrm{XTS}=68$; Cellex $=80)$

$\begin{array}{llll}\text { Yes } & 54(79 \%) & 50(63 \%) & 0.03 \\ \text { No } & 14(21 \%) & 30(37 \%) & \end{array}$

Is the decision to perform an individual ECP procedure at your center using this instrument dependent on patient clinical status? $($ XTS $=66$; Cellex $=76$ )

$\begin{array}{llll}\text { Yes } & 53(80 \%) & 63(83 \%) & \text { NS } \\ \text { No } & 13(20 \%) & 13(17 \%) & \end{array}$

Which of the following clinical findings would prevent a patient from receiving ECP treatment at your center using this instrument? (select all that apply) $(\mathrm{XTS}=53$; Cellex $=63$ )

\begin{tabular}{|c|c|c|}
\hline Hemodynamic instability due to active sepsis & $50(94 \%)$ & $57(90 \%)$ \\
\hline Hemodynamic instability due to heart failure & $41(77 \%)$ & $48(76 \%)$ \\
\hline Positive blood culture in the past $24 \mathrm{~h}$ & $38(72 \%)$ & $38(60 \%)$ \\
\hline Currently febrile & $30(57 \%)$ & $37(59 \%)$ \\
\hline $\begin{array}{l}\text { Bleeding risk to due current anticoagulant } \\
\text { or abnormal coagulation testing }\end{array}$ & $28(53 \%)$ & $27(43 \%)$ \\
\hline $\begin{array}{l}\text { Bleeding requiring red cell or platelet } \\
\text { transfusion in the past } 24 \mathrm{~h}\end{array}$ & $22(42 \%)$ & $20(32 \%)$ \\
\hline IV fat emulsion administration in the past $24 \mathrm{~h}$ & $21(40 \%)$ & $22(35 \%)$ \\
\hline Recent surgery & $19(36 \%)$ & $16(25 \%)$ \\
\hline Dialysis or other fluid shifts in the last $24 \mathrm{~h}$ & $11(21 \%)$ & $11(17 \%)$ \\
\hline Use of ACE-inhibitor in the last $24 \mathrm{~h}$ & $10(19 \%)$ & $11(17 \%)$ \\
\hline \multicolumn{3}{|c|}{ Which anticoagulants are used by your center when performing ECP on this instrument? $(\mathrm{XTS}=68 ;$ Cellex $=80$ ) } \\
\hline Heparin only & $33(48 \%)$ & $25(30 \%)$ \\
\hline ACD-A only & $8(12 \%)$ & $18(22 \%)$ \\
\hline Heparin and/or ACD-A & $27(39 \%)$ & $37(45 \%)$ \\
\hline
\end{tabular}

assessing pediatric ECP will be necessary to obtain meaningful and accurate data on this patient population.

There are several logical future directions that emerge as a result of this study. The rationales, as well as more details about relevant clinical algorithms, regarding thresholds of clinical and laboratory parameters for deferring ECP treatments remain unknown. Based on the institutional heterogeneity suggested by the survey results, there is clinical equipoise in this area that would benefit from future explorations. Regarding characteristics of the ECP buffy coat product, future investigations of performing and analyzing quality control data and relating this back to patient and procedural variables will assist in identifying what parameters are significant, and would help support or refute preprocedure thresholds that are currently in place. To get a better understanding of the volume of procedures being 


\section{Minimum Patient Weight by Device}

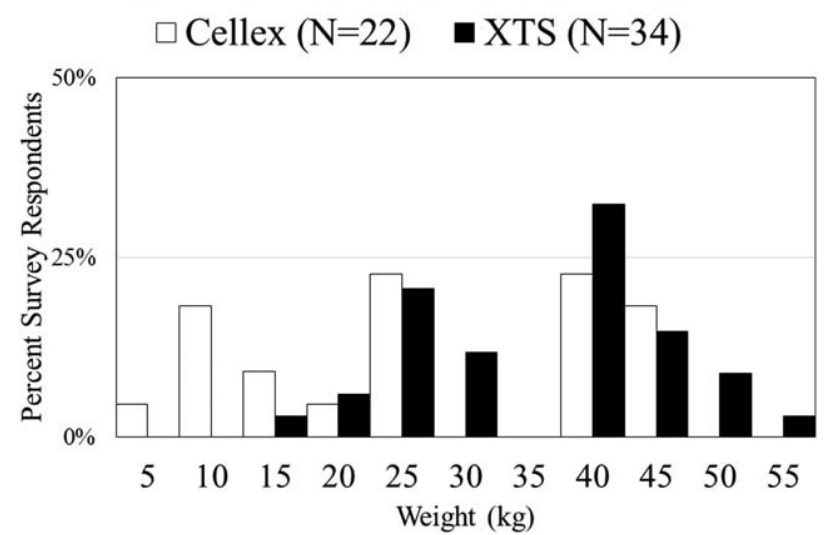

FIG URE 3 Distribution of minimum weight in kilograms for ECP treatment by device. The $x$-axis shows the minimum required weight in kilograms versus the $y$-axis of percent of survey responses reporting this value

performed for specific disease indications, apheresis databases maintained at the participating institutions could be combined; in such a way, higher-volume centers performing ECP for less common indications could be better distinguished. Lastly, investigations targeting geographic areas outside of the United States would help characterize practice patterns in those areas and provide groups for comparison studies.

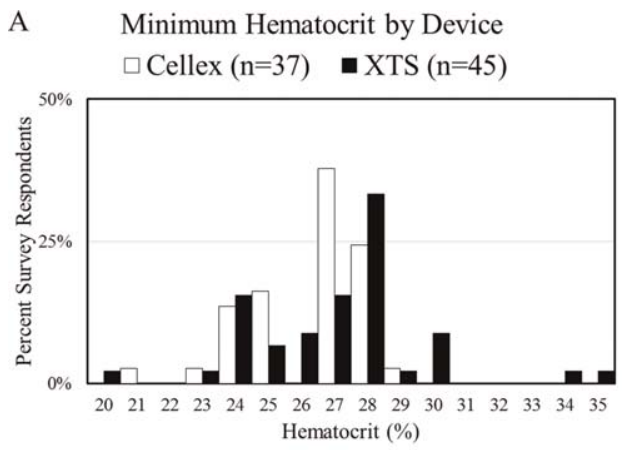

\section{5 | CONCLUSIONS}

We report results of a large survey describing current ECP practice, primarily in North America. Most centers treat small numbers of patients. Centers are providing ECP for diseases that are uncategorized in the 2013 ASFA guidelines based on limited availability of data supporting the use of ECP for these indications. Although not required, a few centers are performing quality control testing on the cellular product prior to re-infusion using a variety of methods. A limited number of ECP devices are currently available, and there are device-specific differences in lower weight limits and laboratory parameters necessary to qualify for ECP. There is a lack of consensus on clinical factors that may warrant deferral of ECP. Further research to support standardization of patient qualification, procedure parameters and product quality assessment is recommended.

\section{SOURCE OF SUPPORT}

None.

\section{CONFLICT OF INTEREST}

None.
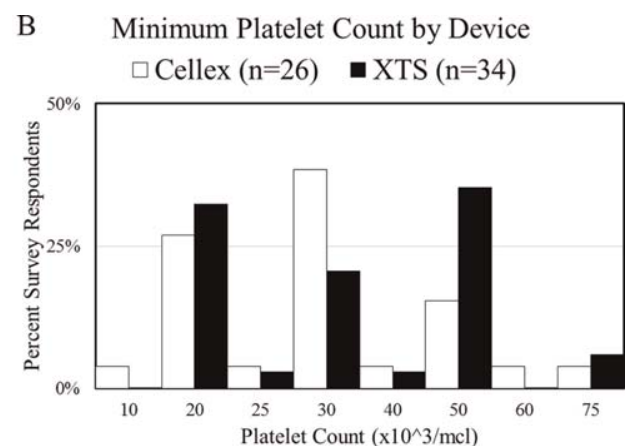

C Minimum White Blood Cell Count by Device $\square$ Cellex (n=19) $\quad$ XTS $(\mathrm{n}=25)$

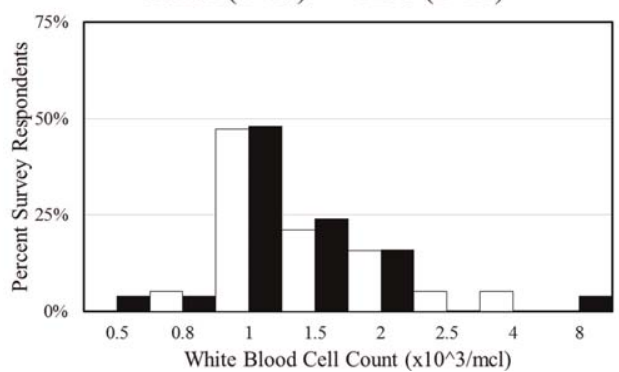

FIGURE 4 Distribution of minimum laboratory test values required for ECP treatment by device. Panel A: The $x$-axis shows the minimum required hematocrit versus the $y$-axis of percent of survey responses reporting this value. Panel B: The $x$-axis shows the minimum required platelet count versus the $y$-axis of percent of survey responses reporting this value. Panel C: The $x$-axis shows the minimum required WBC count versus the $y$-axis of percent of survey responses reporting this value 


\section{REFERENCES}

[1] Ward DM. Extracorporeal photopheresis: how, when, and why. J Clin Apher. 2011;26:276-285.

[2] Marques MB, Adamski J. Extracorporeal photopheresis: technique, established and novel indications. J Clin Apher. 2014;29:228-234.

[3] Edelson RL. Mechanistic insights into extracorporeal photochemotherapy: efficient induction of monocyte-todendritic cell maturation. Transfus Apher Sci. 2014;50: 322-329.

[4] Edelson R, Berger C, Gasparro F, et al. Treatment of cutaneous T-cell lymphoma by extracorporeal photochemotherapy. Preliminary results. N Engl J Med. 1987;316:297-303.

[5] Kapadia E, Wong E, Perez-Albuerne E, Jacobsohn D. Extracorporeal photopheresis performed on the CELLEX ${ }^{\circledR}$ compared with the UVAR-XTS ${ }^{\circledR}$ instrument is more efficient and better tolerated in children with steroid-refractory graft-versus-host disease. Pediatr Blood Cancer. 2015;62: 1485-1488.

[6] Rangarajan HG, Punzalan RC, Camitta BM, Talano JA. The use of novel Therakos $\operatorname{Cellex}(\mathrm{R})$ for extracorporeal photopheresis in treatment of graft-versus-host disease in paediatric patients. Br J Haematol. 2013;163:357-364.

[7] Schwartz J, Winters JL, Padmanabhan A, et al. Guidelines on the use of therapeutic apheresis in clinical practice-evidence-based approach from the Writing Committee of the American Society for Apheresis: the sixth special issue. J Clin Apher. 2013;28:145-284.

[8] Allareddy V, Allareddy V, Konety BR. Specificity of procedure volume and in-hospital mortality association. Ann Surg. 2007;246:135-139.

[9] Allareddy V, Ward MM, Allareddy V, Konety BR. Effect of meeting Leapfrog volume thresholds on complication rates following complex surgical procedures. Ann Surg. 2010;251:377-383.

[10] Yu HY, Hevelone ND, Lipsitz SR, Kowalczyk KJ, Nguyen PL, Hu JC. Hospital volume, utilization, costs and outcomes of robot-assisted laparoscopic radical prostatectomy. J Urol. 2012;187:1632-1637.

[11] Schwartz J, Padmanabhan A, Aqui N, et al. Guidelines on the use of therapeutic apheresis in clinical practiceevidence-based approach from the Writing Committee of the American Society for Apheresis: the seventh special issue. J Clin Apher. 2016;31:149-162.
[12] Bisaccia E, Vonderheid EC, Geskin L. Safety of a new, single, integrated, closed photopheresis system in patients with cutaneous T-cell lymphoma. Br J Dermatol. 2009; 161:167-169.

[13] Schneiderman J, Jacobsohn DA, Collins J, Thormann K, Kletzel M. The use of fluid boluses to safely perform extracorporeal photopheresis (ECP) in low-weight children: a novel procedure. J Clin Apher. 2010;25:63-69.

[14] Kajiume T, Kawano Y, Takaue Y, et al. Continuous monitoring of hematocrit values during apheresis for allogeneic peripheral blood stem cell collection. J Hematother. 1998; 7:493-7.

[15] Carson JL, Grossman BJ, Kleinman S, et al. Red blood cell transfusion: a clinical practice guideline from the AABB. Ann Intern Med. 2012;157:49-58.

[16] Kaufman RM, Djulbegovic B, Gernsheimer T, et al. Platelet transfusion: a clinical practice guideline from the AABB. Ann Intern Med. 2015;162:205-213.

[17] Liu C, Shah K, Dynis M, Eby CS, Grossman BJ. Linear relationship between lymphocyte counts in peripheral blood and buffy coat collected during extracorporeal photopheresis. Transfusion. 2013;53:2635-2643.

[18] Berger M, Albiani R, Sini B, Fagioli F. Extracorporeal photopheresis for graft-versus-host disease: the role of patient, transplant, and classification criteria and hematologic values on outcome-results from a large singlecenter study. Transfusion. 2015;55:736-747.

[19] Nedelcu E, Ziman A, Fernando LP, Cook K, Bumerts P, Schiller G. Exclusive use of acid citrate dextrose for anticoagulation during extracorporeal photopheresis in patients with contraindications to heparin: an effective protocol. J Clin Apher. 2008;23:66-73.

[20] Dyer M, Neal MD, Rollins-Raval MA, Raval JS. Simultaneous extracorporeal membrane oxygenation and therapeutic plasma exchange procedures are tolerable in both pediatric and adult patients. Transfusion. 2014;54:11581165.

[21] Kawai Y, Cornell TT, Cooley EG, et al. Therapeutic plasma exchange may improve hemodynamics and organ failure among children with sepsis-induced multiple organ dysfunction syndrome receiving extracorporeal life support. Pediatr Crit Care Med. 2015;16:366-374.

[22] Sanford KW, Balogun RA. Therapeutic apheresis in critically ill patients. J Clin Apher. 2011;26:249-251. 